\title{
Ceramic materials have similar short term survival rates to other materials on posterior teeth
}

\author{
Abstracted from \\ Pol CW, Kalk W. A \\ Systematic review of ceramic inlays in posterior teeth: an update. \\ Int J Prosthodont. 2011; 24: 566-575. \\ Address for correspondence Dr Warner Kalk, Center for Dentistry and Oral Hygiene, \\ University Medical Center, Groningen, The Netherlands, Antonius Deusinglaan 1, \\ 9713 AV Groningen, The Netherlands. Email:ctm@meg.umcg.nl
}

\section{Question: Do ceramic inlays have better longevity, aesthetic qualities and less postoperative discomfort than other restorative materials for permanent teeth?}

Data sources PubMed, Cochrane and Picarta databases and references of retrieved articles were searched from 2001-2009. Study selection RCTs, CCTs and case series which compared Class I and Class II ceramic inlay restorations in permanent premolar and molar teeth, other posterior restorations were included.

Data extraction and synthesis Two authors reviewed all abstracts independently, compared results and reached consensus on inclusion/ exclusion through discussion. Quality assessment of the studies was carried out using Hayashi's criteria.

Results Three studies (two RCTs, one CCR) were included. All three compared ceramic materials to composite resin materials. The outcomes were longevity of the restorations (USPHS criteria in two studies and CDA in one), postoperative sensitivity and colour match. None of the included studies reported sufficient data to calculate the corrected survival rate, so the conclusion that there was no difference between ceramic and other posterior restorations could not be reappraised. Neither of the two RCTs reporting postoperative sensitivity found a difference between the ceramic or composite restorations confirming the previous reviews findings. For aesthetic quality, only the CCR results were considered sufficiently reliable, with no significant difference being found between the materials. Conclusions Ceramic materials perform as well as alternative restorative materials for use as inlay restorations. However, a lack of long-term data means that this conclusion can only be supported for periods up to one year for longevity and 57 months for colour match.

\section{Commentary}

This paper aims to update the systematic review by Hayashi et al. published in 2003 on the clinical performance of ceramic inlays compared to other restorative materials. ${ }^{1}$ This is an important topic as the information can be used to inform patients as to the likely outcomes of their treatment. The review opens with an excellent and succinct overview of the types of ceramic available to practitioners with reference to their properties and composition.

Three articles were included in this review and all compared ceramic inlays with composite resin. ${ }^{2,3,4}$ This could be seen as disappointing as it would be of interest to know how ceramic performs against more traditional materials such as dental amalgam and gold alloy. In terms of longevity, problems with the methodology of the included articles meant there were insufficient data to calculate corrected survival times and no conclusion could be drawn.

Endodontic complications are an important aspect of any restorative treatment. Details are only given from one of the included studies; three teeth restored with ceramic and four teeth restored with composite out of a total of 135 teeth required root canal treatment over an observation period of up to 58 months. ${ }^{4}$

Ceramic is often recommended to patients as being the most aesthetic treatment option. However, it was concluded that there was no significant difference in the aesthetic result between ceramic inlays and direct composite at up to 58 months . ${ }^{4}$

In summary, the authors state that Hayashi's previous conclusion on porcelain inlays still stands: there is no significant difference in clinical performance between ceramic inlays and other materials at one year. While this review has succeeded in its aim it has added little to our knowledge of the clinical performance of ceramic inlays when compared to other restorative materials. This should not be seen as the fault of the authors; rather that the quality of research published in recent years has not been sufficient to conclusively provide an answer when subjected to rigorous analysis. There is a need for further studies in this area so patients can be more reliably informed as to the likely outcome of their choice of restorative material over time.

\section{Practice points}

- No significant difference in performance of ceramic inlays vs other restorative materials at one year

- No significant difference between the aesthetic of direct composite and ceramic at up to 58 months

- No significant post-operative sensitivity with ceramic inlays

- Data not currently of sufficient quality to make an assessment of ceramic inlays vs other restorative materials at more than one year.

\section{Simon Critchlow}

Department of Restorative Dentistry, Royal London Dental Hospital, New Road, Whitechapel, London, UK.

1. Hayashi M, Wilson NH, Yeung CA, Worthington HV. Systematic review of ceramic inlays. Clin Oral Investig. 2003; 7: 8-19

2. Fasbinder DJ, Dennison JB, Heys DR, Lampe K. The clinical performance of CAD/ CAM-generated composite inlays. J Am Dent Assoc. 2005; 136: 1714-1723

3. Thordrup M, Isidor F, Hörsted-Bindslev P. A prospective clinical study of indirect and direct composite and ceramic inlays: ten year-results. Quintessence Int. 2006; 37: 139-144

4. Lange RT, Pfeiffer P. Clinical evaluation of ceramic inlays compared to composite restorations. Oper Dent. 2009; 34: 263-272

Evidence-Based Dentistry (2012) 13, 49. doi:10.1038/sj.ebd.6400860 